Japanische Hülsenfrucht gegen Krebs

Tolle Bohne oder Humbug?

Frage: Nattokinase aus der japanischen Volksmedizin scheint positive Effekte auf die Blutfließeigenschaften zu haben sowie das Krebsund Osteoporoserisiko zu senken. Was halten Sie davon? Und gibt es Dosierungsempfehlungen?

Prof. Dr. Bern Schultes, Rohrschach: Ich selbst habe keine Erfahrung mit Nattokinase. Wir haben aber ein bisschen recherchiert. Die Nattokinase ist eine Serinprotease, welche von Bacillus natto nach Inkubination mit Sojabohnen gebildet und freigesetzt wird. Das Enzym hat offensichtlich fibrinolytische Aktivität. Kleine, zum Teil unkontrollierte Kurzzeit-Studien haben tatsächliche positive Effekte auf den Blutdruck, Gerinnungssystem und Lipide gezeigt, was einen positiven Effekt bezüglich kardiovaskulärer Erkrankungen suggeriert. Leider liegen meines Wissens nach keine Endpunktstudien oder Langzeit-

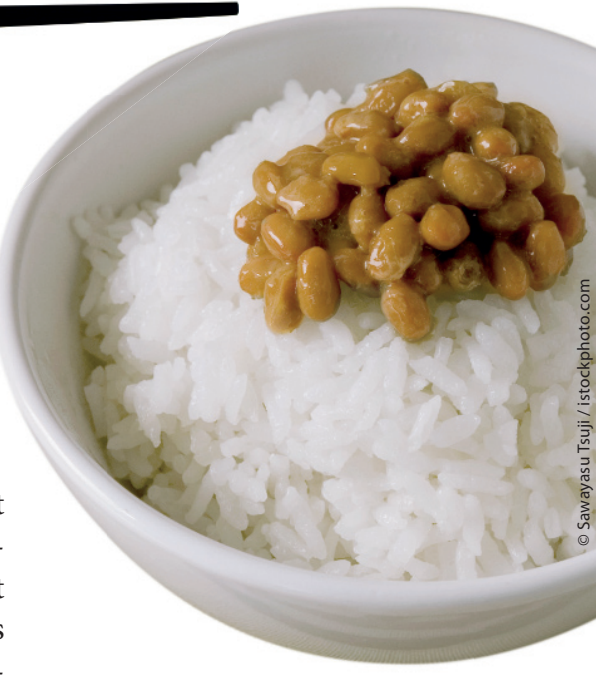

studien vor. Auch über potentielle Interaktion mit Arzneimitteln habe ich keine zuverlässigen Informationen gefunden. Es ist somit aus meiner Sicht eine gewisse Vorsicht geboten. In den Studien wurde meist 2.000 fibrinolytischen Einheiten pro Tag gegeben.

Expertenrat Ernährungsmedizin, www.springermedizin.de

\section{Hochdosis-Singleshot? Cortison bei Colitis}

Frage: Eine Patientin (30 Jahre) am Ende der Stillzeit mit Erstdiagnose Colitis ulcerosa wird aufgrund des Stillens aktuell nur mit Mesalazin behandelt. Auf eine orale Cortisontherapie wurde bislang verzichtet. Vor Jahren habe ich von der guten Effektivität einer Singleshot-Hochdosis-Steroidtherapie gelesen. Wäre dies eine Option?

Dr. Martin Held, München:

Nein, die Singleshot Therapie hat sich nicht etabliert. Sie wird bei der Erstdiagnose einer aktiven Colitis ulcerosa nicht zur Remission führen. Sollten 3 bis 4,5 g Mesalazin nicht ausreichen, bliebe nur Kortison, beginnend mit $60 \mathrm{mg}$ Prednisolon für eine Woche und einer wöchentlichen Dosisreduktion um jeweils $10 \mathrm{mg}$. Nachdem die Patientin am Ende der Stillzeit ist, würde ich zum Abstillen neigen.

Expertenrat Gastroenterologie, www.springermedizin.de

\section{Dr. „Boulevard-Presse“ empfiehlt, aber ... Vertreiben Omega-3- Fettsäuren Depressionen?}

Frage: Eine Patientin mit bipolaraer Störung hat in der Boulevard-Presse über die Behandlung von Depression mit Omega-3-Fettsäuren gelesen. Sie erhofft sich damit "Heilung". Gibt es dafür eine Indikation und existieren seriöse Studien hierzu?

\section{Dr. Johanna Sasse, Chemnitz:}

Nein. Ich weiß zwar von einigen Fachkollegen, die dies propagieren, dennoch gibt es hierzu keine wissenschaftlichen relevanten Studienergebnisse. Ich verweise in diesem Zusammenhang auf die Depressionsleitlinie (DGPPN). Sie sollten dies der Patientin mitteilen und auf die gängigen, bewährten Therapieoptionen, darunter Mood stabilizer (Lithium und Lamotrigin), atypische Neuroleptika (Quetiapin) und im Ausnahmefall die kurzzeitige medikamentöse Therapie mit Antidepressiva verweisen.

Expertenrat Depression, www.springermedizin.de

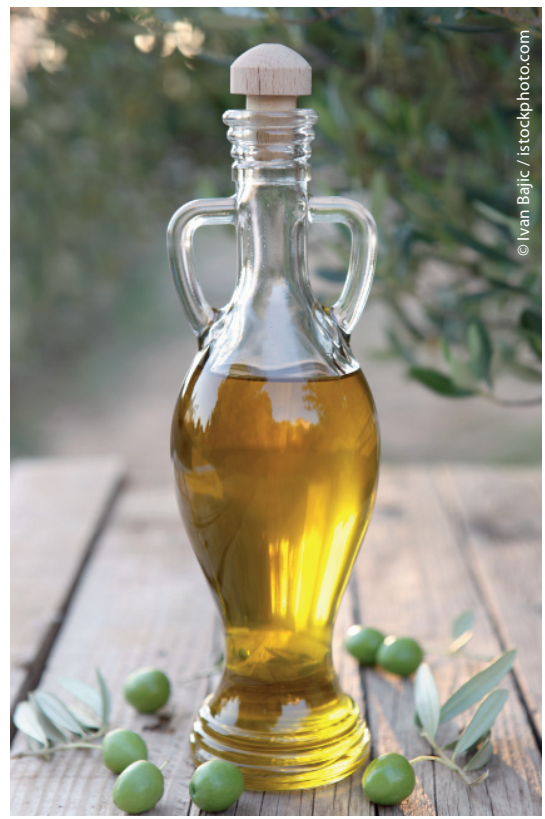

